Ein Jahr Emissionshandel in Kalifornien

\section{Ein Hoffnungsschimmer jenseits des Atlantiks}

\author{
Die Umsetzung des Emissionshandels stellt viele Länder \\ vor erhebliche Probleme. Kalifornien könnte mit \\ seinem neuen Handelssystem neue Standards setzen. \\ Welche Neuerungen beinhaltet das System? \\ Von Sven Rudolph
}

D erzeit plagt sich der Emissionshandel der Europäischen Union (EU) trotz des gerade gestarteten Backloading weiter mit Problemen und auch in anderen Ländern wie Japan und Australien ist die Etablierung anspruchsvoller Kohlenstoffmärkte schwieriger als erwartet. Nachdem Kalifornien, weltweit die neuntgrößte Volkswirtschaft, bereits in der Vergangenheit oft als umweltpolitischer Vorreiter in den USA agiert hat, tut der progressive Westküstenstaat dies nun auch im Klimaschutz. Zwar waren die Neuenglandstaaten bereits 2009 mit einem eng begrenzten, aber immerhin auf vollständiger Versteigerung basierenden Emissionshandel gestartet; nun setzt Kalifornien aber mit einem deutlich umfassenderen und ambitionierteren System neue Standards.

\section{System mit Modellcharakter}

Denn das kalifornische Emissionshandelssystem hat in vielerlei Hinsicht Modellcharakter. Zunächst deckelt es nicht nur die Emissionen aus der Industrie und der Stromerzeugung, sondern bezieht auch Stromimporte ein und ab 2015 sogar den Transport- und Gebäudesektor. Insgesamt fallen dann 85 Prozent der kalifornischen Treibhausgase unter das Cap, ein deutlich größerer Anteil der Gesamtemissionen als in der EU. Zudem muss das kalifornische System mit seinem Reduktionsziel von 17 Prozent bis 2020 (Basis 2012) zumindest auf dem amerikanischen Kontinent als vorbildlich gelten, ist es doch sogar an- spruchsvoller als die oft gelobte British Columbia Carbon Tax. Dass Knappheit an Emissionsrechten herrscht, zeigen dann auch die Preise für Kohlenstoffdioxid $\left(\mathrm{CO}_{2}\right)$ zwischen elf und 15 US Dollar, die nicht nur über dem EU-Niveau liegen, sondern zudem ab 2015 deutlich steigen dürften. Nicht zuletzt verteilt das Programm den gesamten ökonomischen Wert der Emissionsrechte teils über direkte Kompensationen wirtschaftsschwacher Kommunen, teils über Investitionen in Energiesparmaßnahmen und erneuerbare Energien an die Stromkonsumenten zurück, sodass nicht nur Windfall Profits vermieden werden, sondern die Haushalte trotzt Strompreissteigerungen sogar netto profitieren.

Gleichsam gibt es durchaus Raum für Nachbesserungen, die, beobachtet man die Evolution des $\mathrm{CO}_{2}$-Handels in Neuengland oder der EU, sogar reelle politische Erfolgschancen haben dürften. In Kalifornien könnten verschärfte Ziele mit einer Orientierung an den Forderungen des Weltklimarates (minus 25 bis 40 Prozent bis 2020, Basis 1990) zu deutlich höheren Preisen und stärkeren Innovationsanreizen führen; und dem Klimaschutz wäre damit auch besser gedient. Außerdem ist die derzeitige Aufkommensverwendung stark regional fokussiert; Fragen der internationalen Gerechtigkeit werden hingegen ausgeklammert. Nicht zuletzt erscheinen die Regeln für die Anerkennung projektbasierter Emissionsgutschriften äußerst großzügig, was den Innovationsdruck in Kalifornien selbst limitieren kann.
Gleichwohl muss Kalifornien nun seine Vorreiterrolle nutzen, um gemeinsam mit Neuengland andere U.S.-Bundesstaaten von den Vorteilen eines eigenen oder sogar gekoppelten Kohlenstoffmarktes zu überzeugen. Unterstützend wirken könnte dabei der Wettbewerb zwischen den Bundesstaaten um möglichst kostengünstige Wege zur Erreichung neuer Emissionsstandards der obersten U. S.-amerikanischen Umweltbehörde. Auch die Aktivitäten in der bereits 2007 gegründeten Western Climate Initiative (WCI), die neben U. S.-amerikanischen und mexikanischen Bundesstaaten auch kanadische Provinzen umfasste, müssen nach einer zwischenzeitlichen Flaute wiederbelebt werden; die jüngste internationale Verknüpfung des kalifornischen Emissionshandels mit dem von Québec ist hier ein vielversprechender Probelauf.

Aber auch die EU und andere Staaten, die an ambitionierten Emissionshandelssystemen interessiert sind, sollten die Entwicklungen in Kalifornien genau beobachten, denn gerade die Einbeziehung von Stromimporten und die Integration weiterer Sektoren ab 2015, die komplette Rückverteilung des ökonomischen Wertes der Emissionslizenzen sowie die jüngste internationale Verknüpfung sind wichtige Meilensteine auf dem Weg zu einem nachhaltigen globalen Kohlenstoffmarkt. Gerade die gleichzeitige Berücksichtigung ökologischer, ökonomischer und sozialer Aspekte in einem solchen System wird eine zentrale Rolle auch für die gesellschaftliche Akzeptanz eines stärker marktbasierten Klimaschutzes spielen.

\section{Literatur}

Rudolph, S./Kawakatsu, T./Lerch, A. (2013): Regional Market-Based Climate Policy in North America - Efficient, Effective, Fair? California's Cap-and-Trade Program vs. British Columbia's Carbon Tax. MAGKS Joint Discussion Paper Series in Economics No. 45-2013.

AUTOR + KONTAKT

Sven Rudolph ist Associate Professor an der Kyoto University, Japan.

E-Mail: rudolph@econ.kyoto-u.ac.jp 